九州大学学術情報リポジトリ

Kyushu University Institutional Repository

\title{
Lagrangian approach to weakly nonlinear stability of an elliptical flow
}

Fukumoto, Yasuhide

Faculty of Mathematics and Mathematical Research Center for Industrial Technology, Kyushu University

Hirota, Makoto

Japan Atomic Energy Agency

Mie, Youichi

Faculty of Mathematics, Kyushu University

http://hdl. handle. net/2324/17019

出版情報 : MI Preprint Series. 2010-20, 2010-04-28. Faculty of Mathematics, Kyushu University バージョン：

権利関係 : 


\section{Preprint Series}

Kyushu University

The Global COE Program

Math-for-Industry Education \& Research Hub

\section{Lagrangian approach to weakly nonlinear stability of an elliptical flow}

\section{Yasuhide Fukumoto, Makoto Hirota \& Youichi Mie}

MI 2010-20

( Received April 28, 2010 )

Faculty of Mathematics

Kyushu University

Fukuoka, JAPAN 


\title{
Lagrangian approach to weakly nonlinear stability of an elliptical flow
}

\author{
Y. Fukumoto ${ }^{1}$, M. Hirota ${ }^{2}$ and Y. Mie ${ }^{1}$ \\ ${ }^{1}$ Faculty of Mathematics and Mathematical Research Center for Industrial \\ Technology, Kyushu University, 744 Motooka, Nishi-ku, Fukuoka 819-0395, Japan \\ ${ }^{2}$ Japan Atomic Energy Agency, Naka, Ibaraki 311-0193, Japan \\ E-mail: yasuhide@math.kyushu-u.ac.jp
}

\begin{abstract}
Rotating flows with elliptically strained streamlines suffer from a parametric resonance instability between a pair of Kelvin waves whose azimuthal wavenumbers are separated by two. We address the weakly nonlinear evolution of amplitude of three-dimensional Kelvin waves, in resonance, on a flow confined in a cylinder of elliptical cross-section. In a traditional Eulerian approach, derivation of the mean-flow induced by nonlinear interaction of Kelvin waves stands as an obstacle. We show how topological idea, or the Lagrangian approach, facilitates calculation of the wave-induced mean flow. A steady incompressible Euler flow is characterized as a state of the maximum of the total kinetic energy with respect to perturbations constrained to an isovortical sheet, and the isovortical perturbation is handled only in terms of the Lagrangian variables. The criticality in energy of a steady flow allows us to work out the wave-induced mean flow only from the linear Lagrangian displacement. With the mean flow at hand, the Lagrangian approach provides us with a bypass to enter into weakly nonlinear regime of amplitude evolution of three-dimensional disturbances. Unlike the Eulerian approach, the amplitude equations are available directly in the Hamiltonian normal form.
\end{abstract}




\section{Introduction}

It is well known that a vortex tube embedded in a strain flow suffers from threedimensional (3D) instability, being referred to as the Moore-Saffman-Tsai-Widnall (MSTW) instability [17, 20, 3, 5]. The MSTW instability is typically a parametric resonance, driven by the imposed shear, between left- and right-handed helical waves. The waves on a circular cylindrical vortex tube are called the Kelvin waves or the inertial waves. In general, a vortex tube with elliptic core goes through a parametric resonance when two Kelvin waves, with difference in azimuthal wave numbers $m$ being 2, are simultaneously excited. Fukumoto [5] showed on the ground of the Hamiltonian spectral theory that all the intersection points of dispersion curves of the Kelvin waves with $m$ and $m+2$ result in instability. The $(m, m+2)=(1,3)$ and $(0,2)$ resonances were detected in a confined geometry $[4,12]$. Malkus [14] created a rotating flow with strained streamlines in a water-filled flexible cylinder pressed by two stationary rollers (see also [4]). His experiment showed that the MSTW modes grow, followed by excitation of a number of waves and then by eventual disruption. A knowledge of nonlinear growth of linearly unstable modes is indispensable for describing a route to the collapse.

Nonlinear effect comes into play at a matured stage of exponential growth of disturbance amplitude and modifies evolution of the MSTW instability. Waleffe [22] and Sipp [19] showed that the weakly nonlinear effect acts to saturate the amplitude of the Kelvin waves. Mason and Kerswell [15] proceeded to the secondary instability of the MSTW instability. We shall show that their procedure is incomplete in the sense that they did not determine, to the full detail, the mean flow induced by nonlinear interactions of the Kelvin waves. Rodrigues and Luca [18] dealt with the case where mean flow is absent, and found chaotic orbits.

The Lagrangian displacement field is instrumental in handling interaction of waves $[2,9,10]$. Fukumoto and Hirota [7] developed the Lagrangian approach to derive the wave-induced mean flow. The Lagrangian approach allows us to give the mean flow solely in terms of the Lagrangian displacement of first order in amplitude. We rest on this approach to deduce weakly nonlinear amplitude equations. The purpose of this paper is to amend the previous Eulerian treatment and thereby to manipulate the amplitude equations for weakly nonlinear evolution of the MSTW instability. We limit ourselves to the stationary resonance of left- and right-handed helical waves.

In $\S 2$ and 3, we develop the Lagrangian approach to calculate the energy of and the mean flow induced by waves on a steady flow. We recollect the Kelvin waves in $\S 4$ and inquire into the mean flow induced by nonlinear interactions of Kelvin waves in $\S 5$. We recollect the MSTW instability in $\S 6$ and 7 , and enter into the weakly nonlinear regime in $\S 8$. We close with conclusions $(\S 9)$. 


\section{Lagrangian approach}

The signature of the energy of the waves is a key ingredient for the Hamiltonian bifurcation theory. In the presence of a basic flow, the calculation of energy of waves is unattainable in the framework of the traditional approach of using the Eulerian variables. A way out is to use the derivative of the dispersion relation with respect to the frequency [5], but without justification. A steady state of the Euler flows is characterized as an extremal of the kinetic energy with respect to isovortical disturbance [2]. The use of criticality facilitates the calculation of the excess energy by which the kinetic energy increases in the presence of an induced wave. The isovortical disturbance, for which the disturbance vorticity is frozen into the flow, is expressible faithfully in terms of the Lagrangian variables. Mathematical construction of the energy, along with the verification of its relation with the derivative of the dispersion relation, was carried through in our previous papers $[9,10]$. The disturbance velocity of second-order in amplitude suggests the existence of a mean flow [7]. This section presents a brief sketch of a geometric approach to this [8].

Recent studies in the field of weakly nonlinear analyses, sometimes, associated with the wave-mean flow interaction, revealed that the Lagrangian approach of incorporating the topological nature of a fluid is more effective than the traditional Eulerian approach $[2,11]$. Our method $[9,10,7]$, given below, has the same spirits as those studies, though with some novelty in mathematical formulation.

The motion of an inviscid incompressible fluid is regarded as an orbit on $S \operatorname{Diff}(\mathcal{D})$, the group of the volume-preserving diffeomorphisms on the domain $\mathcal{D} \subset \mathbb{R}^{3}$. Its Lie algebra $\mathfrak{g}$ is the velocity field of the fluid. Let $\mathfrak{g}^{*}$ be the dual space of $\mathfrak{g}$ with respect to natural pair $\langle u, v\rangle$ between $u \in \mathfrak{g}$ and $v \in \mathfrak{g}^{*}$. In the context of fluid flows, an element of $\mathfrak{g}^{*}$ is a vector field, and $<,>$ is simply the scalar product between vector fields. The Lie bracket [, ] of $\mathfrak{g}$ is the adjoint representation, which operates on the vector field as

$$
\operatorname{ad}\left(u_{1}\right) u_{2}=\left[u_{1}, u_{2}\right]=\left(\boldsymbol{u}_{2} \cdot \nabla\right) \boldsymbol{u}_{1}-\left(\boldsymbol{u}_{1} \cdot \nabla\right) \boldsymbol{u}_{2} \text { for } u_{1}, u_{2} \in \mathfrak{g},
$$

where the bold symbol is used for clarification of it being a vector field.

Define the Lie-Poisson bracket for functions $F_{1}$ and $F_{2}$ on $\mathfrak{g}^{*}$ by

$$
\left\{F_{1}, F_{2}\right\}=\left\langle\left[\frac{\delta F_{1}}{\delta v}, \frac{\delta F_{2}}{\delta v}\right], v\right\rangle .
$$

The Euler equations for an inviscid incompressible is written in the form of the Poisson equation $\partial F / \partial t=\{F, H\}$. By an introduction of the dual operator ad* of ad by $\left\langle u, \operatorname{ad}(\xi)^{*} v\right\rangle=\langle\operatorname{ad}(\xi) u, v\rangle$ with $\xi \in \mathfrak{g}$, the Poisson equation provides us with an evolution equation for $v \in \mathfrak{g}^{*}[1]$ :

$$
\frac{\partial v}{\partial t}=-\operatorname{ad}^{*}\left(\frac{\delta H}{\delta v}\right) v
$$

If $\delta H / \delta v$ is replaced by an arbitrary $u(t) \in \mathfrak{g},(3)$ is called the Euler-Poincaré equation 
[11]. The operation of $\mathrm{ad}^{*}$ reads in component wise, for the Euler equations,

$$
\left[\operatorname{ad}^{*}(\boldsymbol{\xi}) \boldsymbol{v}\right]_{i}=[-\boldsymbol{\xi} \times(\nabla \times \boldsymbol{v})+\nabla f]_{i},
$$

where $f$ is a function on $\mathcal{D}$ and $\boldsymbol{\xi}$ is a vector field on $\mathcal{D}$. We may identify $\boldsymbol{v} \in \mathfrak{g}^{*}$ as a solenoidal vector field on $\mathcal{D}$ which is tangent to the boundary $\mathcal{D}$ by a suitable adjustment of $f$. The solution of (3) is a coadjoint orbit $v(t)=\operatorname{Ad}^{*}\left(\varphi_{t}^{-1}\right) v(0)$. Here $\varphi_{t}$ is a one-parameter subgroup of $\operatorname{S\operatorname {Diff}}(\mathcal{D})$ generated by $\delta H / \delta v$. The collection of the coadjoint orbit $\left\{\operatorname{Ad}^{*}(\varphi) v(0) \in \mathfrak{g}^{*} \mid \varphi \in S \operatorname{Diff}(\mathcal{D})\right\}$ is alternatively called the isovortical sheet. The velocity field is written by

$$
u\left(t_{0}\right)=\left.\frac{\partial}{\partial t}\right|_{t_{0}}\left(\varphi_{t} \circ \varphi_{t_{0}}^{-1}\right)=\frac{\delta H}{\delta v} .
$$

Given $\varphi_{t}$, an orbit on $S \operatorname{Diff}(\mathcal{D})$, of a basic flow and $v(t)$ as the basic solution in $\mathfrak{g}^{*}$, suppose that $v(0)$ is disturbed to $v_{\alpha}(0)=\operatorname{Ad}^{*}\left(\varphi_{\alpha, 0}^{-1}\right) v(0)$ with $\varphi_{\alpha, 0} \in S \operatorname{Diff}(\mathcal{D})$. Here $\alpha \in \mathbb{R}$ is a small parameter measuring the amplitude of the disturbance field. The disturbed initial condition $v_{\alpha}(0)$ is maintained on the same isovortical sheet as $v(t)$, and so is the subsequent orbit $v_{\alpha}(t)$. It follows that, at each instant $t$, there exists a diffeomorphism $\varphi_{\alpha, t} \in S \operatorname{Diff}(\mathcal{D})$ such that $v(t)$ is disturbed to

$$
v_{\alpha}(t)=\operatorname{Ad}^{*}\left(\varphi_{\alpha, t}^{-1}\right) v(t)=\operatorname{Ad}^{*}\left(\left(\varphi_{\alpha, t} \circ \varphi_{t}\right)^{-1}\right) v(0)
$$

For small values of $\alpha, \varphi_{\alpha, t}$ is a near-identity map, and there exists a generator $\xi_{\alpha}(t) \in \mathfrak{g}$ for it, namely, $\varphi_{\alpha, t}=\exp \xi_{\alpha}(t)$. We expand $\xi_{\alpha}$ in a power series in $\alpha$, to $O\left(\alpha^{2}\right)$, as $\xi_{\alpha}=\alpha \xi_{1}+\alpha^{2} \xi_{2} / 2+\cdots$.

Using $\operatorname{Ad}^{*}\left(\varphi_{\alpha, t}^{-1}\right)=\sum_{n=0}^{\infty}\left[-\operatorname{ad}^{*}\left(\xi_{\alpha}\right)\right]^{n} / n !,(6)$ is expanded as $v_{\alpha}=v+\alpha v_{1}+\alpha^{2} v_{2} / 2+$ $\cdots$;

$$
v_{1}=-\operatorname{ad}^{*}\left(\xi_{1}\right) v, \quad v_{2}=-\operatorname{ad}^{*}\left(\xi_{2}\right) v+\operatorname{ad}^{*}\left(\xi_{1}\right) \operatorname{ad}^{*}\left(\xi_{1}\right) v .
$$

In the language of vector calculus, this is translated into

$$
\boldsymbol{v}_{1}=\mathcal{P}\left[\boldsymbol{\xi}_{1} \times \boldsymbol{\omega}\right], \quad \boldsymbol{v}_{2}=\mathcal{P}\left[\boldsymbol{\xi}_{1} \times\left(\nabla \times\left(\boldsymbol{\xi}_{1} \times \boldsymbol{\omega}\right)\right)+\boldsymbol{\xi}_{2} \times \boldsymbol{\omega}\right],
$$

where $\boldsymbol{\omega}=\nabla \times \boldsymbol{v}$, with identification of $\boldsymbol{v}=v \in \mathfrak{g}^{*}$, and $\mathcal{P}$ is an operator projecting to solenoidal vector field. Likewise, the velocity field of the disturbed orbit $\varphi_{\alpha, t} \circ \varphi_{t}$ is expanded as

$$
\begin{aligned}
u_{\alpha}\left(t_{0}\right) & =\left.\frac{\partial}{\partial t}\right|_{t_{0}}\left(\varphi_{\alpha, t} \circ \varphi_{t} \circ \varphi_{t_{0}}^{-1} \circ \varphi_{\alpha, t_{0}}^{-1}\right) \\
& =u\left(t_{0}\right)+\sum_{n=0}^{\infty} \frac{1}{(n+1) !}\left[\operatorname{ad}\left(\xi_{\alpha}\right)\right]^{n}\left(\frac{\partial \xi_{\alpha}}{\partial t}-\operatorname{ad}(v) \xi_{\alpha}\right) .
\end{aligned}
$$

The first few terms of $u_{\alpha}=u+\alpha u_{1}+\alpha^{2} u_{2} / 2+\cdots$ are

$$
\begin{aligned}
& u_{1}=\frac{\partial \xi_{1}}{\partial t}-\operatorname{ad}(u) \xi_{1} \\
& u_{2}=\frac{\partial \xi_{2}}{\partial t}-\operatorname{ad}(u) \xi_{2}+\operatorname{ad}\left(\xi_{1}\right)\left(\frac{\partial \xi_{1}}{\partial t}-\operatorname{ad}(u) \xi_{1}\right) .
\end{aligned}
$$


A vector field $u_{\alpha} \in \mathfrak{g}$ and its dual $v_{\alpha} \in \mathfrak{g}^{*}$ is made identifiable as the vector field generated by the Hamiltonian $H$,

$$
u_{\alpha}(t)=\left.\frac{\delta H}{\delta v}\right|_{\alpha}(t)=v_{\alpha}(t)
$$

in view of $H=\int_{\mathcal{D}} v_{\alpha}^{2} / 2 \mathrm{~d} V$ with the density of fluid taken as unity.

With this identification, (8) and (10) are combined to yield

$$
\begin{aligned}
& \frac{\partial \boldsymbol{\xi}_{1}}{\partial t}+(\boldsymbol{U} \cdot \nabla) \boldsymbol{\xi}_{1}-\left(\boldsymbol{\xi}_{1} \cdot \nabla\right) \boldsymbol{U}=\boldsymbol{v}_{1}, \\
& \frac{\partial \boldsymbol{\xi}_{2}}{\partial t}+(\boldsymbol{U} \cdot \nabla) \boldsymbol{\xi}_{2}-\left(\boldsymbol{\xi}_{2} \cdot \nabla\right) \boldsymbol{U}+\left(\boldsymbol{u}_{1} \cdot \nabla\right) \boldsymbol{\xi}_{1}-\left(\boldsymbol{\xi}_{1} \cdot \nabla\right) \boldsymbol{u}_{1}=\boldsymbol{v}_{2},
\end{aligned}
$$

with $\boldsymbol{v}_{1}$ and $\boldsymbol{v}_{2}$ provided by (8). The symbol $\boldsymbol{U}=v$ designates the velocity field of the basic flow, and $\boldsymbol{\omega}=\nabla \times \boldsymbol{U}$ is its vorticity. The first-order equation (12) has been well known, but the second-order equation (13) was first derived in our previous paper [7]. As compared with ref [7], this symbolic derivation furnishes a short-cut to reach (13).

\section{Energy and drift current of disturbance field}

The restriction to isovortical disturbances also facilitates the calculation of increased energy, originating from the superposition of disturbance, $H\left(v_{\alpha}\right)=H(v)+\alpha H_{1}+$ $\alpha^{2} H_{2} / 2+\cdots$, in a power series in $\alpha$. For a steady flow $\partial v / \partial t=0$, the first-order term is shown, by use of (7), to vanish

$$
H_{1}=\left\langle\frac{\delta H}{\delta v}, v_{1}\right\rangle=\left\langle\frac{\delta H}{\delta v},-\operatorname{ad}^{*}\left(\xi_{1}\right) v\right\rangle=-\left\langle\xi_{1}, \frac{\partial v}{\partial t}\right\rangle=0,
$$

consistently with the fact that a steady Euler flow is an extremal of the kinetic energy with respect to disturbances constrained on an isovortical sheet $[1,2]$. Then the excess energy is dominated by the second-order term

$$
H_{2}=-\left\langle\xi_{1}, \frac{\partial v_{1}}{\partial t}\right\rangle=\int \boldsymbol{\omega} \cdot\left(\frac{\partial \boldsymbol{\xi}_{1}}{\partial t} \times \boldsymbol{\xi}_{1}\right) \mathrm{d} V
$$

The advantage of the reduced form (15) of the wave energy cannot be overemphasized; (15) dispenses with the solution $\boldsymbol{\xi}_{2}(\boldsymbol{x}, t)$ of (13). The same form as (15) was derived for the wave energy [2], but the second field $\boldsymbol{\xi}_{2}$ had previously gone unnoticed.

Along the same line with the derivation of the energy, we are able to deduce the drift current driven at $O\left(\alpha^{2}\right)$ by nonlinear interaction of waves [8]. For a given $\eta \in \mathfrak{g}$, consider the momentum $J=\langle\eta, v\rangle$ in this direction. By the Hamiltonian Noether theorem, if the Hamiltonian is invariant with respect to the transformation $\exp \eta, J$ is constant. We define $J_{\alpha}=<\eta, v_{\alpha}>$ for the disturbed field $v_{\alpha}$, and calculate the increment of $J_{\alpha}$ relative to $J$ by expanding in powers of $\alpha$ as $J_{\alpha}=<\eta, v>+\alpha J_{1}+\alpha^{2} J_{2} / 2+\cdots$;

$$
\begin{aligned}
& J_{1}=\left\langle\eta, v_{1}\right\rangle=\left\langle\eta,-\operatorname{ad}^{*}\left(\xi_{1}\right) v\right\rangle=\left\langle\xi_{1}, \operatorname{ad}^{*}(\eta) v\right\rangle, \\
& J_{2}=\left\langle\eta, v_{2}\right\rangle=\left\langle\xi_{2}, \operatorname{ad}^{*}(\eta) v\right\rangle+\left\langle\xi_{1}, \operatorname{ad}^{*}(\eta) v_{1}\right\rangle,
\end{aligned}
$$

where use has been made of $(7)$. 
If the basic flow $v(t)$ has a symmetry such that $\operatorname{ad}^{*}(\eta) v=0$, then $J_{1}=0$ and $J_{2}$ becomes

$$
J_{2}=\left\langle\operatorname{ad}(\eta) \xi_{1},-\operatorname{ad}^{*}\left(\xi_{1}\right) v\right\rangle=\int \boldsymbol{\omega} \cdot\left(\boldsymbol{\xi}_{1} \times \mathcal{L}_{\boldsymbol{\eta}} \boldsymbol{\xi}_{1}\right) \mathrm{d} V .
$$

Here $\mathcal{L}_{\boldsymbol{\eta}} \boldsymbol{\xi}_{1}=-\operatorname{ad}(\eta) \xi_{1}$ is the Lie derivative of $\boldsymbol{\xi}_{1}$ with respect to $\boldsymbol{\eta}$. Notably, the excess momentum $J_{2}$ is expressible solely in terms of the first-order quantity $\boldsymbol{\xi}_{1}$.

\section{Kelvin wave}

We briefly recall the Kelvin waves, linearized disturbances of $O(\alpha)$ on the Rankine vortex in a confined geometry. At the outset, we take, as the basic flow, the rigid-body rotation of an inviscid incompressible fluid confined in a cylinder of circular cross-section of unit radius, though later allowance is made for elliptic deformation.

Let us introduce cylindrical coordinates $(r, \theta, z)$ with the $z$-axis along the centerline. Let the $r$ and $\theta$ components of $2 \mathrm{D}$ basic velocity field $\boldsymbol{U}_{0}$ be $U_{0}$ and $V_{0}$, and the pressure $P_{0}$. The suffix 0 signifies that these quantities pertain to the case of circular cross-section. The basic flow is confined to $r \leq 1$, with the velocity field given by

$$
U_{0}=0, \quad V_{0}=r, \quad P_{0}=r^{2} / 2-1 .
$$

Take, as the disturbance field, $\tilde{\boldsymbol{u}}=\alpha \boldsymbol{u}_{01}$. We focus our attention on a normal mode

$$
\boldsymbol{u}_{01}^{(m)}=A_{m}(t) \boldsymbol{u}_{01}^{(m)}(r) \mathrm{e}^{i m \theta} \mathrm{e}^{i k z}, \quad A_{m}(t) \propto \mathrm{e}^{-i \omega_{0} t},
$$

where $A_{m}$ is a complex function of time $t$ and $\omega_{0}$ is frequency. This velocity field represents a Kelvin wave with azimuthal wavenumber $m$ and axial wavenumber $k$. The linearized Euler equations supplies equations for the radial function $\boldsymbol{u}_{01}^{(m)}$ as

$$
\mathcal{L}_{m, k} \boldsymbol{u}_{01}^{(m)}+\nabla p_{01}^{(m)}=\mathbf{0}, \quad \nabla \cdot \boldsymbol{u}_{01}^{(m)}=0
$$

where

$$
\mathcal{L}_{m, k}=\left(\begin{array}{ccc}
-i\left(\omega_{0}-m\right) & -2 & 0 \\
2 & -i\left(\omega_{0}-m\right) & 0 \\
0 & 0 & -i\left(\omega_{0}-m\right)
\end{array}\right)
$$

The solution is found with ease and the radial component is

$$
u_{01}^{(m)}=\frac{i}{\omega_{0}-m+2}\left\{-\frac{m}{r} J_{m}\left(\eta_{m} r\right)+\frac{\omega_{0}-m}{\omega_{0}-m-2} \eta_{m} J_{m+1}\left(\eta_{m} r\right)\right\},
$$

where $\eta_{m}$ is the radial wavenumber, $\eta_{m}^{2}=\left[4 /\left(\omega_{0}-m\right)^{2}-1\right] k^{2}$, and $J_{m}$ is the $m$-th Bessel function of the first kind. The boundary condition $u_{01}^{(m)}=0$ at $r=1$ gives rise to the dispersion relation $[21,16]$

$$
J_{m+1}\left(\eta_{m}\right)=\frac{\left(\omega_{0}-m-2\right) m}{\left(\omega_{0}-m\right) \eta_{m}} J_{m}\left(\eta_{m}\right)
$$

Figure 1 displays the dispersion relation of helical waves $m= \pm 1$. Curves for $m=-1$ are drawn with solid lines, while those for $m=+1$ are drawn with dashed lines. Infinitely many branches emanate from $\left(k, \omega_{0}\right)=(0,1)$ for $m=1$ and from $\left(k, \omega_{0}\right)=(0,-1)$ for $m=-1$. Notice the absence of isolated modes, as opposed to the unbounded case $[5,20]$. 


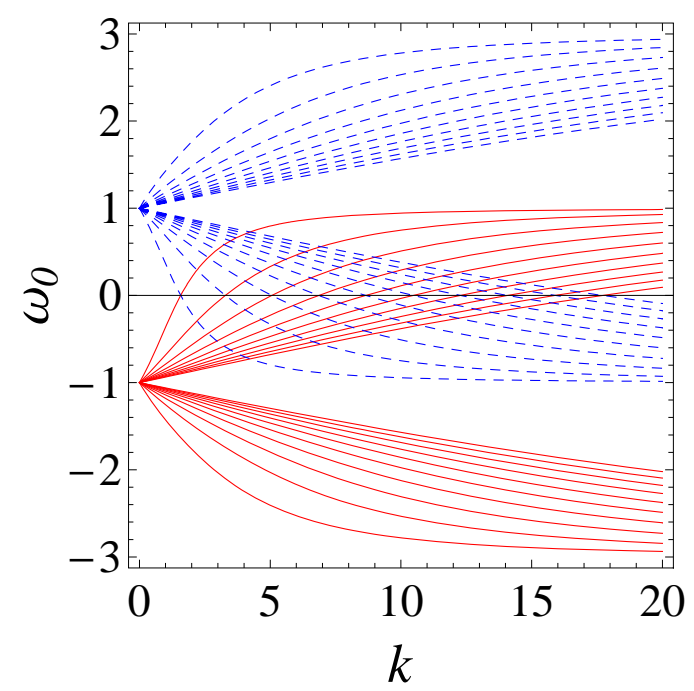

Figure 1. dispersion relation of Kelvin waves, of $m=-1$ (solid lines) and of $m=+1$ (dashed lines), in an elliptic cylinder.

\section{Drift current}

The second-order term $\alpha^{2} \boldsymbol{u}_{02}$ of the disturbance field $\tilde{\boldsymbol{u}}$ includes a mean flow induced by nonlinear interaction of Kelvin waves $\alpha \boldsymbol{u}_{01}$. In keeping with the fact that the basic flow, with circular cylindrical symmetry, admits arbitrary radial profiles of azimuthal and axial velocity. By introducing the elliptical strain of strength $\epsilon$, the functional form of its azimuthal component is somehow manipulated by the solvability condition at $O\left(\epsilon \alpha^{2}\right)$, though limited to intersection points of the dispersion curves [19]. Moreover its coefficient includes an arbitrary parameter, of integration-constant origin, yet to be determined [16].

The Lagrangian approach rescues this difficulty, by producing the mean flow of $O\left(\alpha^{2}\right)$ without having to proceed to a higher order $O\left(\epsilon \alpha^{2}\right)$ [7]. Subsequently, we give a sketch of this Lagrangian approach.

In the context of Kelvin waves, (12) reads

$$
\boldsymbol{u}_{01}=\frac{\partial \boldsymbol{\xi}_{1}}{\partial t}+\left(\boldsymbol{U}_{0} \cdot \nabla\right) \boldsymbol{\xi}_{1}-\left(\boldsymbol{\xi}_{1} \cdot \nabla\right) \boldsymbol{U}_{0}
$$

For our flow $\boldsymbol{U}_{0}=r \boldsymbol{e}_{\theta}$, the RHS of $(24)$ is reduced to $-i\left(\omega_{0}-m\right) \boldsymbol{\xi}_{1}$, and upon substitution from a linear combination of Kelvin waves (19), (24) yields

$$
\boldsymbol{\xi}_{1}=\operatorname{Re}\left[\sum \frac{i A_{m}(t)}{\omega_{0}-m} \boldsymbol{u}_{01}^{(m)}(r) \mathrm{e}^{i m \theta} \mathrm{e}^{i k z}\right],
$$

supplemented by the incompressibility constraint $\nabla \cdot \boldsymbol{\xi}_{1}=0$. Taking the real part should be born in mind.

For the rigid-body rotation $\boldsymbol{U}_{0}=r \boldsymbol{e}_{\theta}$, it is advantageous to calculate the mean flow directly from the spatial average of (8) rather than from (17), since simply $\nabla \times \boldsymbol{U}_{0}=2 \boldsymbol{e}_{z}$. 
It turns out that the second-order field $\boldsymbol{\xi}_{2}$ has no contribution when spatially averaged, leaving

$$
\begin{aligned}
\overline{\boldsymbol{u}_{02}}= & \overline{\mathcal{P}\left[\boldsymbol{\xi}_{1} \times\left(\nabla \times\left(\boldsymbol{\xi}_{1} \times \boldsymbol{e}_{z}\right)\right)\right]}=\overline{\boldsymbol{\xi}_{1} \times \partial \boldsymbol{\xi}_{1} / \partial z} \\
& =\sum \frac{4 i k}{\left(\omega_{0}-m\right)^{2}}\left|A_{m}\right|^{2}\left(0, u_{01}^{(m)} w_{01}^{(m)},-u_{01}^{(m)} v_{01}^{(m)}\right) .
\end{aligned}
$$

The Lagrangian approach is capable of calculating the mean flow at any points satisfied by the dispersion relation $\left(k, \omega_{0}\right)$.

\section{Stability of rotating flow in an elliptic cylinder: setting of problem}

Rotating flows in an elliptic cylinder go through excitation of a number of growing waves, resulting in disruption $[14,4]$. We express elliptic cross-section as

$$
\frac{x^{2}}{1+\epsilon}+\frac{y^{2}}{1-\epsilon}=1 \text {. }
$$

The parameter $\epsilon$ designates the elliptic distortion. We assume that $|\epsilon|$ is small. In conjunction with this distortion the basic flow is perturbed as

$$
\begin{gathered}
\boldsymbol{U}=\boldsymbol{U}_{0}+\epsilon \boldsymbol{U}_{1}+\cdots, \quad P=P_{0}+\epsilon P_{1}+\cdots ; \\
U_{1}=-r \sin 2 \theta, \quad V_{1}=-r \cos 2 \theta, \quad P_{1}=0 .
\end{gathered}
$$

The subscript designates order in elliptic parameter $\epsilon$. The augmented term of $O(\epsilon)$ represents a steady quadrupole field. In other words, $\boldsymbol{U}_{1}$ corresponds to the field consisting of strain field whose stretching direction lies along $\theta=-\pi / 4$ and whose direction of contraction is along $\theta=\pi / 4$.

We add three-dimensional disturbance field $\tilde{\boldsymbol{u}}$ to this two-dimensional basic flow. We consider asymptotic expansions of the velocity field in two small parameters $\epsilon$ and $\alpha$ as

$$
\boldsymbol{u}=\boldsymbol{U}+\tilde{\boldsymbol{u}}=\boldsymbol{U}_{0}+\epsilon \boldsymbol{U}_{1}+\alpha \boldsymbol{u}_{01}+\epsilon \alpha \boldsymbol{u}_{11}+\alpha^{2} \boldsymbol{u}_{02}+\alpha^{3} \boldsymbol{u}_{03}+\cdots,
$$

specifically to $O\left(\alpha^{3}\right)$ in amplitude. Here, the velocity field $\boldsymbol{u}_{m n}$ occurs at $O\left(\epsilon^{m} \alpha^{n}\right)$. The side wall (27) of the cylinder is $r=1+\epsilon \cos 2 \theta / 2+O\left(\epsilon^{2}\right)$ when the elliptic strain $\epsilon$ is small. The boundary condition to be imposed at the rigid side wall is

$$
\boldsymbol{u} \cdot \boldsymbol{n}=0 \quad \text { at } r=1+\epsilon \cos 2 \theta / 2,
$$

where $\boldsymbol{n}$ is the unit outward normal vector to the cylinder boundary.

\section{Moore-Saffman-Tsai-Widnall instability}

The Kelvin waves described in $§ 4$, neutrally stable oscillations, are made unstable, by breaking the circular symmetry of the cylinder cross-section. We explore the effect of elliptic strain $\epsilon \boldsymbol{U}_{1}$ upon the Kelvin waves. If the given disturbance flow, in the absence of elliptic strain, has Kelvin waves with $\mathrm{e}^{i m \theta}$ and $\mathrm{e}^{i(m+2) \theta}$, interaction of these waves via the strain (28), at $O(\epsilon \alpha)$, through the convective terms of the Euler equations excite 
again Kelvin waves with $\mathrm{e}^{i m \theta}$ and $\mathrm{e}^{i(m+2) \theta}$. This coincidence indicates occurrence of parametric instability $[17,20,5]$. Fukumoto [5] made a thorough analysis of the 3D instability of the Rankine vortex embedded in a plane shear field, in an unbounded space, and showed that the parameter resonance instability occurs, at $O(\epsilon \alpha)$, at all intersection points $(k, \omega)$ of dispersion curves of the $m$ and $m+2$ waves. The same is true for the rotating flow confined in an elliptic cylinder [21].

There are intersection points on the $k$-axis $\left(\omega_{0}=0\right)$ at certain values of $k$ as is observed in Figure 1. For left and right-handed helical wave resonance, the stationary mode $\omega_{0}=0$ has far greater growth rate than non-stationary modes $\left(\omega_{0} \neq 0\right)[20,3,5]$. We limit ourselves to the stationary parametric resonance between left and right-handed helical waves $(m, m+2)=(-1,+1)$ occurring at $\omega_{0}=0$.

Under the restriction of $\omega_{0}=0$, the radial wavenumber becomes $\eta=\sqrt{3} k$. Then, we send the following disturbance velocity $\boldsymbol{u}_{01}$, a superposition of $m= \pm 1$ waves,

$$
\boldsymbol{u}_{01}=A_{-} \boldsymbol{u}_{01}^{(-)} \mathrm{e}^{-i \theta} \mathrm{e}^{i k z}+A_{+} \boldsymbol{u}_{01}^{(+)} \mathrm{e}^{i \theta} \mathrm{e}^{i k z}+\text { c.c. }
$$

We use the notation $A_{ \pm}$in place of $A_{ \pm 1}$. Excited at $O(\epsilon \alpha)$ fueled by $\boldsymbol{U}_{1}$ is

$$
\begin{aligned}
\boldsymbol{u}_{11} & =\left\{B_{-} \boldsymbol{u}_{11}^{(-)} \mathrm{e}^{-i \theta}+B_{+} \boldsymbol{u}_{11}^{(+)} \mathrm{e}^{i \theta}+B_{-3} \boldsymbol{u}_{11}^{(-3)} \mathrm{e}^{-3 i \theta}+B_{3} \boldsymbol{u}_{11}^{(3)} \mathrm{e}^{3 i \theta}\right\} \mathrm{e}^{i k z} \\
& + \text { c.c. }
\end{aligned}
$$

The radial functions $\boldsymbol{u}_{11}^{(m)}(r)$ and $\boldsymbol{u}_{11}^{(m+2)}(r)$ are determined by solving inhomogeneous linear ordinary differential equations, derived from the Euler equations and by the continuity equations, subject to the boundary condition (30) at $O(\epsilon \alpha)$,

$$
u_{11}-u_{01} \cos 2 \theta / 2+v_{01} \sin 2 \theta=0 .
$$

The boundary condition (33) provides algebraic equations for $B_{ \pm}$and the solvability condition gives rise to, with the help of the dispersion relation (23),

$$
\frac{1}{A_{+}} \frac{\partial A_{-}}{\partial t_{10}}=\frac{-1}{A_{-}} \frac{\partial A_{+}}{\partial t_{10}}=i \frac{3\left(3 k^{2}+1\right)}{8\left(2 k^{2}+1\right)}=i a,
$$

where $t_{10}=\epsilon t$, the slow time scale, and $k$ is the solution of dispersion relation $J_{1}(\eta)=-\eta J_{0}(\eta)$.

The degenerate modes with $\omega_{0}=0$ necessarily result in parametric resonance with growth rate $a=3\left(3 k^{2}+1\right) /\left[8\left(2 k^{2}+1\right)\right][21]$ and with amplitude ratio of the eigen-function given by $A_{-} / A_{+}=i$. Numerical values of the growth rate are, for a first few intersection points with $\omega_{0}=0,(k, \sigma) \approx(1.578,0.5311),(3.286,0.5542), \cdots$. The occurrence of parametric resonance implies the existence of negative-energy waves. This is indeed the case [5]. The energy of Kelvin waves is efficiently calculated from the formula (15) built in the Lagrangian framework [7].

At $O\left(\alpha^{3}\right)$, the modes $\mathrm{e}^{ \pm i \theta} \mathrm{e}^{i k z}$ again arise, which invites the compatibility conditions. The function $\boldsymbol{u}_{03}^{(m)}$ with $m= \pm 1$ is governed by $\mathcal{L}_{m, k} \boldsymbol{u}_{03}^{(m)}=\mathcal{N}-\partial \boldsymbol{u}_{01}^{(m)} / \partial t_{02}$, with $t_{02}=\alpha^{2} t$. Since the matrix $\mathcal{L}_{m, k}$ is singular, $\left(\partial / \partial t_{02}\right) \boldsymbol{u}_{01}^{(m)}$ is adjusted for the forcing terms to satisfy the solvability condition. The calculation of $\mathcal{N}$ requires the precise form of the mean flow of $O\left(\alpha^{2}\right)$. 


\section{Amplitude equation}

We are now ready to derive the weakly nonlinear amplitude equations to $O\left(\alpha^{3}\right)$. The procedure is described at length in ref [16].

The mean flow induced by nonlinear interactions of helical waves is

$$
4 i k\left(0,\left(\left|A_{-}\right|^{2}+\left|A_{+}\right|^{2}\right) u_{01}^{(+)} w_{01}^{(+)},\left(\left|A_{-}\right|^{2}-\left|A_{+}\right|^{2}\right) u_{01}^{(+)} v_{01}^{(+)}\right) .
$$

For general $(m, m+2)$ parametric resonance, only the radial component of mean flow is zero as is seen from (26). But, in the case of stationary helical-wave parametric resonance, the axial components of mean flow is zero, because $\left|A_{-}\right|=\left|A_{+}\right|[5,19]$. Taking account of this, the boundary condition at $O\left(\alpha^{3}\right)$ leads us immediately to the amplitude equations

$$
\frac{\mathrm{d} A_{ \pm}}{\mathrm{d} t}=\mp i\left[\epsilon a A_{\mp}+\alpha^{2} A_{ \pm}\left(b\left|A_{ \pm}\right|^{2}+c\left|A_{\mp}\right|^{2}\right)\right],
$$

where $a$ is defined by (34) and

$$
\begin{aligned}
b= & \frac{-2 k^{4}}{3\left(2 k^{2}+1\right)}\left[\frac{4}{J_{0}(\eta)^{2}} \int_{0}^{1} r J_{0}(\eta r)^{2} J_{1}(\eta r)^{2} \mathrm{~d} r-\left(11 k^{4}+13 k^{2}+5\right) J_{0}(\eta)^{2}\right] \\
c= & \frac{k^{2}}{12\left(2 k^{2}+1\right)}\left[\frac{64 k^{2}}{J_{0}(\eta)^{2}} \int_{0}^{1} r J_{0}(\eta r)^{2} J_{1}(\eta r)^{2} \mathrm{~d} r\right. \\
& \left.+\left(20 k^{6}+97 k^{4}+14 k^{2}-27\right) J_{0}(\eta)^{2}\right] .
\end{aligned}
$$

By virtue of availability of compact form, these coefficients are readily calculated at all the intersection points on the $k$-axis $\left(\omega_{0}=0\right)$. For the longest two wavelengths, we have $(k ; a, b, c) \approx(1.579 ; 0.5312,-0.3976,5.222),(3.286 ; 0.5542,-8.286,53.39)$.

It is remarkable that the resulting equations (36) are exactly Hamiltonian normal form [13]. The normal form shows up at once with nonlinear terms $\left|A_{ \pm}\right|^{2} A_{ \pm},\left|A_{\mp}\right|^{2} A_{ \pm}$ fully incorporating the effect of mean flow (26). On the other hand, in the Eulerian treatment, the amplitude of the mean flow had to be introduced as an intervening dependent parameter, and its equation produces an undetermined constant [19].

As $A_{-}$and $A_{+}$are complex functions of $t$, the amplitude equations (36) constitute a four-dimensional dynamical system. The sign of coefficients $(a, b, c)$ is unchanged, regardless of the choice of the intersection points: $a>0, b<0$ and $c>0$.

The amplitude equations (36) admits restriction of the phase space to a twodimensional subspace with $A=A_{-}=-A_{+}^{*}$, where $*$ stands for the complex conjugate. The complex amplitude equations (36) collapses, by a choice of $\alpha^{2}=\epsilon$, to

$$
\frac{\mathrm{d} A}{\mathrm{~d} t}=i \epsilon\left(-a A^{*}+\beta|A|^{2} A\right)
$$

where $\beta=b+c$. Figure 2 illustrates the trajectory in the phase space $(\operatorname{Re}[A], \operatorname{Im}[A])$. The rigidly rotating state (the origin) is unstable, but the amplitude of the orbit necessarily saturates within a basin of the unstable equilibrium. 


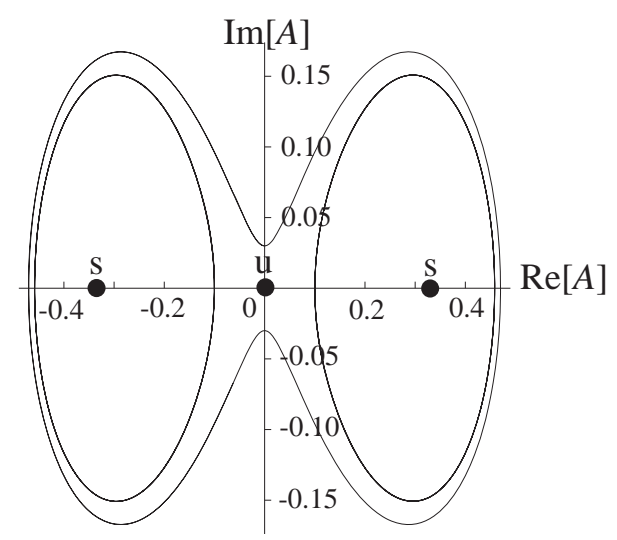

Figure 2. Trajectories in the phase space $(\operatorname{Re}[A], \operatorname{Im}[A])$ for $k=1.579$. The dots designate equilibria (s: stable, u: unstable).

Let us set $A=|A| \mathrm{e}^{i \phi}$. The modulus $|A|$ and the phase $\phi$ satisfy the following equations,

$$
\frac{\mathrm{d}|A|}{\mathrm{d} t}=-\epsilon a|A| \sin 2 \phi, \quad \frac{\mathrm{d} \phi}{\mathrm{d} t}=-\epsilon a \cos 2 \phi+\epsilon \beta|A|^{2} .
$$

The linear effect predominates over the nonlinear effect for small disturbance amplitude $|A|(\ll 1)$. In case the equilibrium point $A=0$ is unstable, the direction of disturbance vorticity $\phi$ is liable to be parallel to the unstable direction $\phi=-\pi / 4$. The elliptic strain makes horizontal vortex lines continuously stretched, if they are oriented, on average, in the direction of $\phi=-\pi / 4$. This is the mechanism for the MSTW instability at the linear stage. When the disturbance grows substantially, $|A| \approx 1$ say, the nonlinear effect is called into play. In view of (39), the nonlinear effect is exclusively rotating the phase angle $\phi$. As a consequence, alignment of horizontal vorticity to the direction $\phi=-\pi / 4$ is hindered, which renders the disturbance amplitude saturate.

The compact form of the coefficients (37) makes it feasible to manipulate the short-wavelength asymptotics of equilibrium amplitude [16]. The short-wavelength asymptotics of (36) is found without difficulty, and amplitude of the saturated state is found to be $|A|_{\mathrm{eq}}=\sqrt{a / \beta} \rightarrow 3 / 4\left(\sqrt{3} \pi /\left(k^{3} \log k\right)\right)^{1 / 2}$.

\section{Conclusion}

We have made a weakly nonlinear analysis of the short-wave instabilities of rotating flow in a cylinder of elliptic cross-section. We put emphasis on the advantage of the Lagrangian approach, over the Eulerian one, in the derivation of the mean flow induced by nonlinear interactions of the Kelvin waves $(\S 5)$.

The phase of the complex amplitude of $O(\alpha)$ of the 3D disturbance represents the angle, from the $x$-axis, of oscillating vorticity disturbances in the horizontal plane. For small amplitude, the features of the linear short-wave instability is retrieved that selectively amplification of the particular is invited, for which the disturbance vorticity is 
continuously stretched by the ambient strain $[20,5]$. The non-linear effect suppresses this monotonic growth by turning the disturbance vorticity out of the stretching direction as soon as the amplitude becomes sufficiently large [19].

However this behavior does not coincide with the vigorous amplification of a number of waves and the ultimate disruption of a strained flow observed in experiments $[14,4]$. This indicates that the nonlinear interaction of a single MSTW mode is far from sufficient in describing practical flows. The secondary and the tertiary instability, which may be invited before reaching the stage of nonlinear saturation, will drastically alter the subsequent evolution $[15,6]$. The Lagrangian approach would be vital for dealing with these higher-order bifurcations.

\section{Acknowledgments}

We are grateful to Yuji Hattori for invaluable suggestions. Y. F. was supported in part by a Grant-in-Aid for Scientific Research from the Japan Society for the Promotion of Science (Grant No. 21540390).

\section{References}

[1] Arnol'd V I 1966 Ann. Inst. Fourier Grenoble 16319

[2] Arnol'd V I 1966 J. Méc. 529

[3] Eloy C and Le Dizès S 2001 Phys. Fluids 13660

[4] Eloy C, Le Gal P and Le Dizès S 2000 Phys. Rev. Lett. 853400

[5] Fukumoto Y 2003 J. Fluid Mech. 493287

[6] Fukumoto Y, Hattori Y and Fujimura K 2005 Proc. of the 3rd International Conference on Vortex Flows and Vortex Models (The Japan Society of Mechanical Engineers) p 149

[7] Fukumoto Y and Hirota M 2008 Phys. Scr. T132 014041

[8] Hirota M 2009 Math-for-Industry Tutorial: Spectral theories of non-Hermitian operators and their application COE Lecture Note Vo. 20 (Kyushu University) p.137

[9] Hirota M and Fukumoto Y 2008 J. Math. Phys. 49083101

[10] Hirota M and Fukumoto Y 2008 Phys. Plasmas 15122101

[11] Holm D D, Schmah T and Stoica C 2009 Geometric Mechanics and Symmetry (Oxford University Press)

[12] Kerswell R R 2002 Annu. Rev. Fluid Mech. 3483

[13] Knobloch E, Mahalov A and Marsden J E 1994 Physica D 7349

[14] Malkus W V R 1989 Geophys. Astrophys. Fluid Dyn. 48123

[15] Mason D M and Kerswell R R 1999 J. Fluid Mech. 39673

[16] Mie Y and Fukumoto Y 2010 J. Math-for-Industry 227

[17] Moore D W and Saffman P G 1975 Proc. R. Soc. Lond. A 346413

[18] Rodrigues S B and Luca J D 2009 Phys. Fluids 21014108

[19] Sipp D 2000 Phys. Fluids 121715

[20] Tsai C-Y and Widnall S E 1976 J. Fluid Mech. 73721

[21] Vladimirov V A, Tarasov V F and Rybak L Y 1983 Izv. Atmos. Ocean. Phys. 19437

[22] Waleffe F A 1989 PhD thesis. MIT 


\section{List of MI Preprint Series, Kyushu University}

The Global COE Program

Math-for-Industry Education \& Research Hub

MI

MI2008-1 Takahiro ITO, Shuichi INOKUCHI \& Yoshihiro MIZOGUCHI

Abstract collision systems simulated by cellular automata

MI2008-2 Eiji ONODERA

The intial value problem for a third-order dispersive flow into compact almost Hermitian manifolds

MI2008-3 Hiroaki KIDO

On isosceles sets in the 4-dimensional Euclidean space

MI2008-4 Hirofumi NOTSU

Numerical computations of cavity flow problems by a pressure stabilized characteristiccurve finite element scheme

MI2008-5 Yoshiyasu OZEKI

Torsion points of abelian varieties with values in nfinite extensions over a padic field

MI2008-6 Yoshiyuki TOMIYAMA

Lifting Galois representations over arbitrary number fields

MI2008-7 Takehiro HIROTSU \& Setsuo TANIGUCHI

The random walk model revisited

MI2008-8 Silvia GANDY, Masaaki KANNO, Hirokazu ANAI \& Kazuhiro YOKOYAMA Optimizing a particular real root of a polynomial by a special cylindrical algebraic decomposition

MI2008-9 Kazufumi KIMOTO, Sho MATSUMOTO \& Masato WAKAYAMA

Alpha-determinant cyclic modules and Jacobi polynomials 
MI2008-10 Sangyeol LEE \& Hiroki MASUDA

Jarque-Bera Normality Test for the Driving Lévy Process of a Discretely Observed Univariate SDE

MI2008-11 Hiroyuki CHIHARA \& Eiji ONODERA

A third order dispersive flow for closed curves into almost Hermitian manifolds

MI2008-12 Takehiko KINOSHITA, Kouji HASHIMOTO and Mitsuhiro T. NAKAO

On the $L^{2}$ a priori error estimates to the finite element solution of elliptic problems with singular adjoint operator

MI2008-13 Jacques FARAUT and Masato WAKAYAMA

Hermitian symmetric spaces of tube type and multivariate Meixner-Pollaczek polynomials

MI2008-14 Takashi NAKAMURA

Riemann zeta-values, Euler polynomials and the best constant of Sobolev inequality

MI2008-15 Takashi NAKAMURA

Some topics related to Hurwitz-Lerch zeta functions

MI2009-1 Yasuhide FUKUMOTO

Global time evolution of viscous vortex rings

MI2009-2 Hidetoshi MATSUI \& Sadanori KONISHI

Regularized functional regression modeling for functional response and predictors

MI2009-3 Hidetoshi MATSUI \& Sadanori KONISHI

Variable selection for functional regression model via the $L_{1}$ regularization

MI2009-4 Shuichi KAWANO \& Sadanori KONISHI

Nonlinear logistic discrimination via regularized Gaussian basis expansions

MI2009-5 Toshiro HIRANOUCHI \& Yuichiro TAGUCHII

Flat modules and Groebner bases over truncated discrete valuation rings 
MI2009-6 Kenji KAJIWARA \& Yasuhiro OHTA

Bilinearization and Casorati determinant solutions to non-autonomous $1+1$ dimensional discrete soliton equations

\section{MI2009-7 Yoshiyuki KAGEI}

Asymptotic behavior of solutions of the compressible Navier-Stokes equation around the plane Couette flow

MI2009-8 Shohei TATEISHI, Hidetoshi MATSUI \& Sadanori KONISHI

Nonlinear regression modeling via the lasso-type regularization

MI2009-9 Takeshi TAKAISHI \& Masato KIMURA

Phase field model for mode III crack growth in two dimensional elasticity

MI2009-10 Shingo SAITO

Generalisation of Mack's formula for claims reserving with arbitrary exponents for the variance assumption

MI2009-11 Kenji KAJIWARA, Masanobu KANEKO, Atsushi NOBE \& Teruhisa TSUDA Ultradiscretization of a solvable two-dimensional chaotic map associated with the Hesse cubic curve

\section{MI2009-12 Tetsu MASUDA}

Hypergeometric T -functions of the q-Painlevé system of type $E_{8}^{(1)}$

MI2009-13 Hidenao IWANE, Hitoshi YANAMI, Hirokazu ANAI \& Kazuhiro YOKOYAMA A Practical Implementation of a Symbolic-Numeric Cylindrical Algebraic Decomposition for Quantifier Elimination

MI2009-14 Yasunori MAEKAWA

On Gaussian decay estimates of solutions to some linear elliptic equations and its applications

MI2009-15 Yuya ISHIHARA \& Yoshiyuki KAGEI

Large time behavior of the semigroup on $L^{p}$ spaces associated with the linearized compressible Navier-Stokes equation in a cylindrical domain 
MI2009-16 Chikashi ARITA, Atsuo KUNIBA, Kazumitsu SAKAI \& Tsuyoshi SAWABE Spectrum in multi-species asymmetric simple exclusion process on a ring

MI2009-17 Masato WAKAYAMA \& Keitaro YAMAMOTO

Non-linear algebraic differential equations satisfied by certain family of elliptic functions

MI2009-18 Me Me NAING \& Yasuhide FUKUMOTO

Local Instability of an Elliptical Flow Subjected to a Coriolis Force

MI2009-19 Mitsunori KAYANO \& Sadanori KONISHI

Sparse functional principal component analysis via regularized basis expansions and its application

MI2009-20 Shuichi KAWANO \& Sadanori KONISHI

Semi-supervised logistic discrimination via regularized Gaussian basis expansions

MI2009-21 Hiroshi YOSHIDA, Yoshihiro MIWA \& Masanobu KANEKO

Elliptic curves and Fibonacci numbers arising from Lindenmayer system with symbolic computations

MI2009-22 Eiji ONODERA

A remark on the global existence of a third order dispersive flow into locally Hermitian symmetric spaces

MI2009-23 Stjepan LUGOMER \& Yasuhide FUKUMOTO

Generation of ribbons, helicoids and complex scherk surface in laser-matter Interactions

MI2009-24 Yu KAWAKAMI

Recent progress in value distribution of the hyperbolic Gauss map

MI2009-25 Takehiko KINOSHITA \& Mitsuhiro T. NAKAO

On very accurate enclosure of the optimal constant in the a priori error estimates for $H_{0}^{2}$-projection 
MI2009-26 Manabu YOSHIDA

Ramification of local fields and Fontaine's property (Pm)

MI2009-27 Yu KAWAKAMI

Value distribution of the hyperbolic Gauss maps for flat fronts in hyperbolic three-space

MI2009-28 Masahisa TABATA

Numerical simulation of fluid movement in an hourglass by an energy-stable finite element scheme

MI2009-29 Yoshiyuki KAGEI \& Yasunori MAEKAWA Asymptotic behaviors of solutions to evolution equations in the presence of translation and scaling invariance

MI2009-30 Yoshiyuki KAGEI \& Yasunori MAEKAWA

On asymptotic behaviors of solutions to parabolic systems modelling chemotaxis

MI2009-31 Masato WAKAYAMA \& Yoshinori YAMASAKI

Hecke's zeros and higher depth determinants

MI2009-32 Olivier PIRONNEAU \& Masahisa TABATA

Stability and convergence of a Galerkin-characteristics finite element scheme of lumped mass type

MI2009-33 Chikashi ARITA

Queueing process with excluded-volume effect

MI2009-34 Kenji KAJIWARA, Nobutaka NAKAZONO \& Teruhisa TSUDA

Projective reduction of the discrete Painlevé system of type $\left(A_{2}+A_{1}\right)^{(1)}$

MI2009-35 Yosuke MIZUYAMA, Takamasa SHINDE, Masahisa TABATA \& Daisuke TAGAMI Finite element computation for scattering problems of micro-hologram using DtN map 
MI2009-36 Reiichiro KAWAI \& Hiroki MASUDA

Exact simulation of finite variation tempered stable Ornstein-Uhlenbeck processes

MI2009-37 Hiroki MASUDA

On statistical aspects in calibrating a geometric skewed stable asset price model

MI2010-1 Hiroki MASUDA

Approximate self-weighted LAD estimation of discretely observed ergodic OrnsteinUhlenbeck processes

MI2010-2 Reiichiro KAWAI \& Hiroki MASUDA

Infinite variation tempered stable Ornstein-Uhlenbeck processes with discrete observations

MI2010-3 Kei HIROSE, Shuichi KAWANO, Daisuke MIIKE \& Sadanori KONISHI Hyper-parameter selection in Bayesian structural equation models

MI2010-4 Nobuyuki IKEDA \& Setsuo TANIGUCHI

The Itô-Nisio theorem, quadratic Wiener functionals, and 1-solitons

MI2010-5 Shohei TATEISHI \& Sadanori KONISHI

Nonlinear regression modeling and detecting change point via the relevance vector machine

MI2010-6 Shuichi KAWANO, Toshihiro MISUMI \& Sadanori KONISHI

Semi-supervised logistic discrimination via graph-based regularization

MI2010-7 Teruhisa TSUDA

UC hierarchy and monodromy preserving deformation

MI2010-8 Takahiro ITO

Abstract collision systems on groups

MI2010-9 Hiroshi YOSHIDA, Kinji KIMURA, Naoki YOSHIDA, Junko TANAKA \& Yoshihiro MIWA

An algebraic approach to underdetermined experiments 
MI2010-10 Kei HIROSE \& Sadanori KONISHI

Variable selection via the grouped weighted lasso for factor analysis models

MI2010-11 Katsusuke NABESHIMA \& Hiroshi YOSHIDA

Derivation of specific conditions with Comprehensive Groebner Systems

MI2010-12 Yoshiyuki KAGEI, Yu NAGAFUCHI \& Takeshi SUDOU

Decay estimates on solutions of the linearized compressible Navier-Stokes equation around a Poiseuille type flow

MI2010-13 Reiichiro KAWAI \& Hiroki MASUDA

On simulation of tempered stable random variates

MI2010-14 Yoshiyasu OZEKI

Non-existence of certain Galois representations with a uniform tame inertia weight

MI2010-15 Me Me NAING \& Yasuhide FUKUMOTO

Local Instability of a Rotating Flow Driven by Precession of Arbitrary Frequency

MI2010-16 Yu KAWAKAMI \& Daisuke NAKAJO

The value distribution of the Gauss map of improper affine spheres

MI2010-17 Kazunori YASUTAKE

On the classification of rank 2 almost Fano bundles on projective space

MI2010-18 Toshimitsu TAKAESU

Scaling limits for the system of semi-relativistic particles coupled to a scalar bose field

MI2010-19 Reiichiro KAWAI \& Hiroki MASUDA

Local asymptotic normality for normal inverse Gaussian Lévy processes with high-frequency sampling

MI2010-20 Yasuhide FUKUMOTO, Makoto HIROTA \& Youichi MIE

Lagrangian approach to weakly nonlinear stability of an elliptical flow 